\title{
Determining Atomic Structures from Digitally Defined Regions of Nanocrystals
}

\author{
M Gallagher-Jones ${ }^{1}$, K Bustillo ${ }^{2}$, C Ophus ${ }^{2}$, L Richards ${ }^{1}$, J Ciston ${ }^{2}, \mathrm{~S} \mathrm{Lee}^{3}$, A Minor $^{4}$, J Rodriguez ${ }^{1}$ \\ ${ }^{1}$ UCLA, Los angeles, CA, ${ }^{2}$ Lawrence Berkeley National Laboratory, Berkeley, CA, ${ }^{3}$ Sungkyunkwan \\ University, Suwon, Korea, Republic of, ${ }^{4}$ University of California Berkeley, Berkeley, CA \\ marcusgj@chem.ucla.edu
}

Crystallography is intrinsically limited by its reliance on signal averaged over large collections of perfectly ordered molecules within a single crystal lattice or even across multiple crystals. This is particularly pronounced when crystals contain severe disorder pathologies or are beam sensitive. Recent advances in x-ray and electron diffraction have reduced the minimum size of crystals useful for structure determination to 100 s of nms 1-3, overcoming many of these difficulties. We previously applied 4-dimensional scanning transmission electron microscopy (4D-STEM), a scanning diffraction technique, to the study of nanomosaicity within a single micron-scale crystal4. This highlighted a potential opportunity for using scanning nano-beam diffraction for structure determination. In our current work, we extend developments in 4D-STEM by incorporating tomography to solve atomic structures of macromolecules from specific regions of polymer nanocrystals. Scanning nanobeam electron diffraction tomography (nanoEDT) consists of scanning a sub-10nm electron probe over a peptide nanocrystal whilst rotating the crystal in discrete, 1-degree intervals5. At each tilt angle, a 4D-STEM dataset is collected by a direct electron detector, capturing thousands of sparse diffraction patterns mapped to specific locations within the crystal. The use of direct electron detection, in combination with cryogenic data collection and a hybrid counting algorithm, allows even weak signals from high-resolution Bragg peaks to be accurately recorded from radiation-sensitive crystals. This intensity data, representing an angular wedge of reciprocal space, is extracted from computationally defined regions of the scan and used to compute structures via fragment-based phasing methods. By scanning the beam, we can collect diffraction from a wide field-of-view and digitally recombine it for later analysis, obviating the need for a selected area aperture. Data collected by nanoEDT compares favourably with data acquired under more conventional electron diffraction methodologies (microED), with minimal evidence of radiation damage. NanoEDT breaks new ground in nanocrystallography by allowing atomic structures to be determined from any region of a nanocrystal through the use of virtual apertures, potentially leading to the determination of atomic structures from heterogeneous or polycrystalline nanoassemblies. References 1. Lanza, A. et al. Nanobeam precession-assisted 3D electron diffraction reveals a new polymorph of hen egg-white lysozyme. IUCrJ 6, (2019). 2. Mugnaioli, E. et al. $\mathrm{Ab}$ Initio Structure Determination of $\mathrm{Cu} 2-\mathrm{x}$ Te Plasmonic Nanocrystals by Precession-Assisted Electron Diffraction Tomography and HAADF-STEM Imaging. Inorganic chemistry 57, 10241-10248 (2018). 3. Sawaya, $\mathrm{M}$. R. et al. Ab initio structure determination from prion nanocrystals at atomic resolution by MicroED. PNAS 113, 11232-11236 (2016). 4. Gallagher-Jones, M. et al. Nanoscale mosaicity revealed in peptide microcrystals by scanning electron nanodiffraction. Communications Biology 2, 26 (2019). 5. Gallagher-Jones, M. et al. Atomic structures determined from digitally defined nanocrystalline regions. IUCrJ 7, 3 (2020)

Acta Cryst. (2020). A76, a111 